\title{
91. Notes on Knots and Periodic Transformations
}

\author{
By Shin'ichi KInoshita \\ Department of Mathematics, Osaka University \\ (Comm. by K. KunUGI, M.J.A., July 12, 1957)
}

Introduction. Let $T$ be a sense preserving periodic transformation of the 3-sphere $S^{3}$ onto itself. Furthermore let $T$ be different from the identity and have at least one fixed point. Then it has been shown by Smith $^{9)}$ that the set $F$ of all fixed points of $T$ is a simple closed curve. Recently Montgomery, Zippin and Samelson ${ }^{536)}$ have studied about the position of $F$ in $S^{3}$, which also be concerned in this note. Hereafter we always assume that $T$ is semilinear, and then $F$ is polygonal. Let $p$ be the period of $T$. Identifying the points

$$
x, T(x), \cdots, T^{p-1}(x)
$$

in $S^{3}$, we have an orientable 3-manifold $M$. Then it will be proved in $\S 4$ that $M$ is simply connected, i.e. the fundamental group of $M$ consists of only one element. In $\$ 5$, under the assumption that the well-known Poincaré conjecture on 3-manifolds is true, we shall prove that almost all knots of the Alexander-Briggs's table ${ }^{1)}$ are not equivalent to $F$, if $T$ is of period 2 . This will be done by the use of Alexander polynomials. ${ }^{2)}$ To prove these we shall study knots in 3manifolds in $\$ \S 1-3$. In this note everything will be considered from the semilinear point of view.

$\S 1$. Let $M$ be a compact 3-manifold (without boundary) and $k$ an oriented simple closed curve in $M$. The fundamental group of $M-k$ will be denoted by $F(M-k)$ or sometimes by $F(k, M)$. Hereafter we always assume that $k$ is homologous to 0 in $M$. Let $V$ be a sufficiently small tubular neighbourhood of $k$ in $M$. Then the boundary $\dot{V}$ of $V$ is a torus. A meridian of $\dot{V}$ is a simple closed curve on $\dot{V}$ which bounds a 2-cell in $V$ but not on $\dot{V}$. Let $x$ be an oriented meridian of $\dot{V}$. Since $k$ is homologous to 0 in $M$, the linking number Link $(k, x)$ of $k$ and $x$ can be defined and is equal to \pm 1 . We may always suppose that $x$ is oriented such that

$$
\text { Link }(k, x)=1 \text {. }
$$

For each integer $p(\neq 0) x^{p}$ is not homotopic to 1 .

Now let $\left\{x, X_{1}, X_{2}, \cdots, X_{n}\right\}$ be the set of generators of $F(M-k)$. Put

and

$$
\operatorname{Link}\left(k, X_{i}\right)=v(i) \quad(i=1,2, \cdots, n)
$$

$$
x_{i}=x^{-v(i)} X_{i} . \quad(i=1,2, \cdots, n)
$$


Then $\left\{x, x_{1}, x_{2}, \cdots, x_{n}\right\}$ forms again the set of generators of $F(M-k)$ and for each $i$

$$
\operatorname{Link}\left(k, x_{i}\right)=0 \text {. }
$$

Let $R_{s}=1(s=1,2, \cdots, m)$ be defining relations of $F(M-k)$ with respect to $\left\{x, x_{i}\right\}$. Then the symbol

$$
\left\{x, x_{1}, \cdots, x_{n}: R_{1}, \cdots, R_{m}\right\}
$$

will be called a presentation ${ }^{3)}$ of $F(M-k)$. A presentation of $F(M)$ is given by

$$
\left\{x, x_{1}, \cdots, x_{n}: x, R_{1}, \cdots, R_{m}\right\} .
$$

§ 2. Let $w \in F^{\prime}(k, M)$. Then $w$ is written as a word which consists of at most $x, x_{1}, \cdots, x_{n}$. Let $f(w)$ be an integer which is equal to the exponent sum of $w$, summed over the element $x$. By (1) it is easy to see that $f$ is a homomorphism of $F(k, M)$ onto the set of all integers. Now put

$$
F_{g}(k, M)=\{w \in F(k, M) \mid f(w)=0(\bmod g)\},
$$

where $g>0$. Then $F_{g}(k, M)$ is a normal subgroup of $F(k, M)$. Therefore there exists uniquely the $g$-fold cyclic covering space $\tilde{M}_{\theta}(k)^{7)}$ of $M-k$, whose fundamental group is isomorphic to $F_{g}(k, M)$. Since $x$ is a meridian of $\dot{V}$, we can also define the $g$-fold cyclic covering space $M_{g}(k)$ of $M$, branched along $k$. For each $g M_{g}(k)$ is a closed 3manifold.

$F\left(\tilde{M}_{g}(k)\right)$ and $F\left(M_{g}(k)\right)$ are calculated from $F(k, M)$ as follows: Let (2) be a presentation of $F(k, M)$. Put

$$
x_{i j}=x^{j} x_{i} x^{-j} \cdot \quad\left(\begin{array}{l}
i=1,2, \cdots, n \\
j=0,1, \cdots, g-1
\end{array}\right)
$$

Since $f\left(R_{s}\right)=0$ for every $s(s=1,2, \cdots, m), x^{j} R_{s} x^{-j}$ is expressible by a word which consists of at most $x_{i j}$ and $x^{g}$. We denote it by notations

Then

$$
x^{j} R_{s} x^{-j}=\widetilde{R}_{s} \text {. }
$$

$$
\left\{x^{g}, x_{i j}: \widetilde{R}_{s}\right\}
$$

is a presentation of $F\left(\widetilde{M}_{g}(k)\right)$ and

is one of $F\left(M_{g}(k)\right)$.

$$
\left\{x^{q}, x_{i j}: x^{g}, \widetilde{R}_{s}\right\}
$$

There is a homomorphism of $F\left(M_{g}(k)\right)$ onto $F(M)$. To prove this: let (3) and (5) be presentations of $F(M)$ and $F\left(M_{g}(k)\right)$, respectively. Put $h\left(x_{i j}\right)=x_{i}$ for each $x_{i j}$. It is easy to see that $h$ can be extended to a homomorphism of $F\left(M_{g}(k)\right)$ onto $F(M)$.

From the above fact we have immediately the following

Theorem 1. Let $M$ be a 3-manifold and $k$ a simple closed curve in $M$ which is homologous to 0 in $M$. If a g-fold cyclic covering space $M_{g}(k)$ of $M$, branched along $k$, is simply connected, then $M$ is also simply connected. 
$\S 3$. Let (2) be a presentation of $F(k, M)$. Put

$$
x^{j} x_{i}^{ \pm 1} x^{-j}= \pm x^{j} x_{i} \quad\left(\begin{array}{l}
i=1,2, \cdots, n \\
j=0, \pm 1, \pm 2, \cdots
\end{array}\right)
$$

and replace the multiplication by the addition. Furthermore suppose that the addition is commutative. Then for each relation $R_{s}=1$ $(s=1,2, \cdots, m)$ we have a relation $\bar{R}_{s}=0$, which is a linear equation of $x_{i}$. If $m<n$, then we add $n-m$ trivial equations $0=0$ to the system of equations and then we may assume that $m \geqq n$. From these linear equations we can make the Alexander matrix, whose $(s, i)$-th term is the coefficient of $x_{i}$ in $\bar{R}_{s}=0$.

If two oriented knots $k_{1}$ and $k_{2}$ in $M$ are equivalent each other, then $F\left(k_{1}, M\right)$ and $F\left(k_{2}, M\right)$ are directly isomorphic. ${ }^{2}$ It was proved by Alexander ${ }^{2)}$ that if two indexed groups are directly isomorphic each other, then the elementary factors different from unity of the Alexander matrices and also their products $\Delta(x, k, M)$ are the same each other. Of course they are determined up to factors $\pm x^{p}$. It should be remarked that $A\left(x, k, M_{g}(k)\right)$ is also defined from (4) replacing $x^{g}$ by $x$.

It can be proved that

$$
\Delta\left(x, k, M_{g}(k)\right)=\dot{\Pi}_{j=0}^{g-1} \Delta\left(\sqrt[g]{x} \omega_{j}, k, M\right),
$$

where $\omega_{j}=\cos \frac{2 \pi j}{g}+i \sin \frac{2 \pi j}{g}$. This is known for the case $M=S^{3}{ }^{4}$ ) But the proof of the latter depends essentially only on the following equation of determinants:

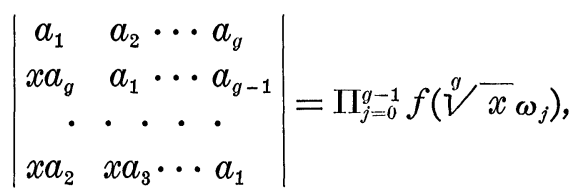

where $f(y)=a_{1}+a_{2} y+\cdots+a_{g} y^{g-1}$. Therefore the proof of our case is the same as the case $M=S^{3}$ and is omitted. As a special case of (6) we have

$$
\Delta\left(1, k, M_{g}(k)\right)=\Pi_{j=0}^{g-1} \Delta\left(\omega_{j}, k, M\right) .
$$

$\Delta\left(1, k, M_{g}(k)\right) \neq 0$ if and only if the 1-dimensional Betti number $p_{1}\left(M_{g}(k)\right)$ $=0$. If $p_{1}\left(M_{g}(k)\right)=0$, then $\left|\Delta\left(1, k, M_{g}(k)\right)\right|$ is equal to the product of torsion numbers (in this case if $\left|\Delta\left(1, k, M_{g}(k)\right)\right|=1$, then $M_{g}(k)$ has no torsion number).

$\S 4$. Now let $T$ be a sense preserving (of course semilinear) periodic transformation of $S^{3}$ onto itself. Furthermore let $T$ be different from the identity and have at least one fixed point. Then the set $F$ of all fixed points of $T$ is a simple closed curve. ${ }^{9}$ Suppose that $p$ is the minimal number of the set of all positive periods of $T$. 
It is easy to see that $T$ is primitive. ${ }^{10)}$ Identifying the points

$$
x, T(x), \cdots, T^{p-1}(x)
$$

in $S^{3}$, we have an orientable 3-manifold $M$. Hereafter we always use $M$ only in this meaning. Since $F$ is homologous to 0 in $S^{3}, F$ is homologous to 0 in $M$. $T$ acts locally as a rotation about $F .^{5)}$ From this it follows that $S^{3}$ is the p-fold cyclic covering space of $M$, branched along $k$. Then by Theorem 1 we have the following

Theorem 2. Suppose that $T$ and $M$ have the above meaning. Then $M$ is simply connected.

$\S 5$. Now we assume that the Poincaré conjecture is true. Then by Theorem $2 M$ is a 3-sphere, and the coefficients of $\Delta(x, F, M)$ are symmetric. ${ }^{8) 111}$ We consider in $\$ 5$ only the case $p=2$.

Suppose first that the degree of $\Delta\left(x, F, S^{3}\right)$ is 2 . Then by (6) the degree of $\Delta(x, F, M)$ is also 2. Put

$$
\Delta(x, F, M)=a x^{2}+b x+a,
$$

where $a \neq 0$ and we may assume that $2 a+b=1$. Then by (6)

$$
\Delta\left(x, F, S^{3}\right)=a^{2} x^{2}+\left(2 a^{2}-b^{2}\right) x+a^{2} .
$$

Furthermore $4 a^{2}-b^{2}= \pm 1$, which means that $2 a-b= \pm 1$. From this it follows that $2 a=1$ or $2 a=0$. Since $a \neq 0$ and $a$ is an integer, this is a contradiction. Thus we have proved that if the degree of $\Delta\left(x, k, S^{3}\right)$ is 2 , then $k$ is not equivalent to $F$.

By the same way it can be seen easily that if the degrees of $\Delta\left(x, F, S^{3}\right)$ are 4,6 and 8 , then $\Delta\left(x, F, S^{3}\right)$ are confined to the following forms, respectively:

$$
\begin{aligned}
& a^{2} x^{4}+2 a(1-2 a) x^{3}+\left(1-4 a+6 a^{2}\right) x^{2}+\cdots, \\
& a^{2} x^{6}-\left(2 a^{2}+b^{2}\right) x^{5}-\left(a^{2}+2 b-4 b^{2}\right) x^{4} \\
& \quad+\left(4 a^{2}-1+4 b-6 b^{2}\right) x^{3}-\cdots, \\
& a^{2} x^{8}+\left(2 a c-b^{2}\right) x^{7}+\left(2 a-4 a c-4 a^{2}+c^{2}+2 b^{2}\right) x^{6} \\
& \quad+\left(2 c-2 a c-4 c^{2}+b^{2}\right) x^{5}+(1-4 a-4 c+8 a c \\
& \left.\quad+6 a^{2}+6 c^{2}-4 b^{2}\right) x^{4}+\cdots .
\end{aligned}
$$

From this we have the following

Theorem 3. Let $T$ be the same as that of Theorem 2. Furthermore suppose that the period of $T$ is 2 . Then, under the assumption that the Poincaré conjecture is true, all knots of the Alexander-Briggs's table, ${ }^{122}$ except for the cases $8_{9}$ and $8_{20}$, are not equivalent to $F$.

Remark. If we do not assume that the Poincaré conjecture is true, then we have the following exceptional case:

$$
\Delta\left(x, F, S^{3}\right)=a^{2} x^{2}-\left(2 \alpha^{2}+1\right) x+a^{2},
$$

even if the degree of $\Delta\left(x, F, S^{3}\right)$ is 2 . The exceptional cases of higher degrees will be more complicated. 


\section{References}

1) J. W. Alexander and G. B. Briggs: Ann. Math., 28, 562-586 (1927).

2) J. W. Alexander: Trans. Amer. Math. Soc., 30, 275-306 (1928).

3) R. H. Fox: Ann. Math., 59, 196-210 (1954).

4) R. H. Fox: Ann. Math., 64, 407-419 (1956).

5) D. Montgomery and H. Samelson: Can. J. Math., 7, 208-220 (1955).

6) D. Montgomery and L. Zippin: Proc. Amer. Math. Soc., 5, 460-465 (1954).

7) H. Seifert and W. Threlfall: Lehrbuch der Topologie (1934).

8) H. Seifert: Math. Ann., 110, 571-592 (1935).

9) P. A. Smith: Ann. Math., 40, 690-711 (1939).

10) P. A. Smith: Appendix $B$ in Lefschetz, Algebraic Topology (1942).

11) G. Torres and R. H. Fox: Ann. Math., 59, 211-218 (1954). 